\title{
HYDROSTATIC PRESSURE EFFECT ON OXYGEN PRECIPITATES IN SILICON SINGLE CRYSTAL
}

\author{
A. Misiuk, J. Adamczewska \\ Institute of Electron Technology \\ Al. Lotników 32/46, 02-668 Warszawa, Poland
}

\section{J. BĄK-MISIUK}

Institute of Physics, Polish Academy of Sciences, Warszawa, Poland

\author{
J. HÄRTWIG \\ Department of Physics, Jena University, Jena, Germany
}

\section{A. MoraWski and Z. WitczaK}

High Pressure Research Centre, Polish Academy of Sciences, Warszawa, Poland

The effect of hydrostatic pressure on some properties of $\mathrm{Cz}-\mathrm{Si}$ with oxygen precipitates is investigated. The observed phenomena are discussed in terms of misfit between the precipitates and $\mathrm{Si}$ matrix.

PACS numbers: 61.70.At, 61.70.-r, 81.40.-z

Czochralski-grown Si single crystals ( $\mathrm{Cz}-\mathrm{Si})$ subjected to hydrostatic pressure (HP) can change defect structure and so the bulk properties influenced by defects. This effect is related mostly to the oxygen-induced defects existing in the bulk of annealed samples [1-4], e.g. oxygen precipitates (OP) composed of $\mathrm{SiO}_{2}$ [3].

In this paper we investigate the effect of $\mathrm{HP}$ on the defect structure of $\mathrm{Cz}-\mathrm{Si}$ monocrystals containing OP. The isostatic and pulsating HP as well as ultrasound (US) treatments at room temperature were applied. Also the high pressure-high temperature (HP-HT) treatment was used to modify the elastic misfit $\varepsilon$ at the $\mathrm{OP} / \mathrm{Si}$ boundary [3].

The OP density $d_{\mathrm{OP}}$ in the preliminary annealed Cz-Si single crystals (oxy-

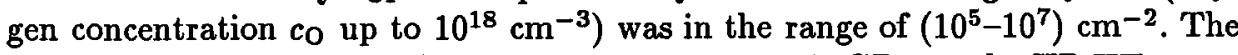
samples were subjected to the HP treatment up to $1.8 \mathrm{GPa}$, to the HP-HT treatment at HT up to $1620 \mathrm{~K}$ or to the HP-US treament at HP up to $1 \mathrm{GPa}$ (the US amplitude up to $9 \mathrm{MPa}$, frequency $17 \mathrm{kHZ}$ ) [5]. The HP induced changes of 
the defect structure were detected by the Lang topography. The bulk properties sensitive to the defect structure (X-ray anomalous transmission $I_{\mathrm{A}}$, lattice constant $a$, linear thermal expansion coefficient $\alpha$ ) were measured by X-ray methods. Measurements were performed after removing the sample from the HP chamber.

In the case of samples HP treated at room temperature the detectable changes of $a_{293 \mathrm{~K}}$ were observed for $d_{\mathrm{OP}}>10^{4} \mathrm{~cm}^{-2}$, being more pronounced for pulsating HP. For example, $a_{293 \mathrm{~K}}=(0.54308870 \pm 0.00000072) \mathrm{nm}$ for the sample with $d_{\mathrm{OP}}=8 \times 10^{5} \mathrm{~cm}^{-2}$ subjected to 12 cycles $0.25-1.8 \mathrm{GPa}$, whereas for the initial HP untreated sample $a_{293 \mathrm{~K}}=(0.54308652 \pm 0.00000074) \mathrm{nm}$. The $I_{\mathrm{A}}$ value diminishes with HP ( $\sim 15 \%$ for $1 \mathrm{GPa})$. Contrary to that, the HP-US treatment causes a decrease of $a$ and slight increase of $I_{\mathrm{A}}$. Annealing of the preliminary HP treated ( 5 cycles at $0.25-0.8 \mathrm{GPa}$ ) samples $\left(d_{\mathrm{OP}} \approx 10^{7} \mathrm{~cm}^{-2}\right)$ at $1370 \mathrm{~K}$ for 20 hours caused the increase of $a$ and decrease of $I_{\mathrm{A}}\left(\Delta a_{293 \mathrm{~K}}\right.$ was about $6 \times 10^{-6} \mathrm{~nm}$ and $I_{\mathrm{A}}$ decreased down to $25 \%$ of the initial value).

Under HP-HT additional defects are created, but smaller OP can dissolve in the matrix at highest HT $[4,6]$. The values of $a$ diminish for the lower HP-HT, but increase $\left(\Delta a_{293 \mathrm{~K}}\right.$ up to $\left.15 \times 10^{-6} \mathrm{~nm}\right)$ for the higher HP-HT treated samples. Thermal expansion coefficient $\alpha$ tends to diminish with HT [4], whereas $I_{\mathrm{A}}$ is rising for the samples HP treated at higher $T$, being dependent also on $d_{\mathrm{OP}}$.

The following phenomena are typical for the HP treated samples:

- the lattice constant $a$ value rises, $I_{\mathrm{A}}$ diminishes.

- after additional annealing this tendency is even more pronounced.

Initial $\mathrm{Cz}-\mathrm{Si}$ single crystals contained $\mathrm{OP}$ in the form of platelets composed of amorphous $\mathrm{SiO}_{2}$ as the main kind of defects. Let us consider for simplicity the spherical shape of OP. Let as assume also that the misfit $\varepsilon_{0}$ at the OP/Si boundary in initial samples is dependent only on the temperature of the final stage of sample preparation (1200-1450 K) and can be estimated taking into account different thermal expansion of $\mathrm{SiO}_{2}$ and $\mathrm{Si}[3,4]$. The relative volume change of the crystal $\Delta V / V$ is connected with the change of lattice parameter $\Delta a / a$ by the following equation:

$$
\Delta a / a=\Delta V / 3 V
$$

During the preparation of initial samples the concentration of interstitial oxygen $c_{\mathrm{O}}$ diminishes to $c_{\mathrm{f}}$. So $\left(c_{\mathrm{O}}-c_{\mathrm{f}}\right) V$ oxygen atoms are in the form of OP. The mean concentration of OP $c_{\mathrm{OP}}$ would be $\left(c_{\mathrm{O}}-c_{\mathrm{f}}\right) / n$, where $n$ means the number of $\mathrm{O}$ atoms in OP. In the equilibrium conditions the averaged dilatation of the Si lattice does not depend on the degree of the oxygen precipitation [7]. However, in our case $\Delta \mathrm{V}$ is not equal to 0 because some misfit was induced during preparation of the initial samples at cooling, being connected with different thermal expansion of $\mathrm{Si}$ and $\mathrm{SiO}_{2}$. This misfit can be changed after the HP-(HT,US) treatment because of additional defects creation. The difference of the OP volume $v_{O P}$ and of the $\mathrm{Si}$ volume $v_{\mathrm{Si}}$ of the same initial volume $v_{0}$, related to the misfit created as the result of the HP treatment, would be $[3,4,6]$ :

$$
\Delta v=v_{\mathrm{OP}}-v_{\mathrm{Si}}=\varepsilon^{\mathrm{c}}\left(4 G / \kappa_{\mathrm{OP}}+3\right) v_{0}=1 / 2\left[\varepsilon^{\mathrm{c}}\left(4 G / \kappa_{\mathrm{OP}}+3\right) n v_{\mathrm{SiO}_{2}}\right],
$$

where $\varepsilon^{c}$ - effective misfit after the HP treatment related also to additionally 
created defects (Fig. 1), $G$ - shear modulus of $\mathrm{Si}, \kappa_{\mathrm{OP}}-$ bulk modulus of $\mathrm{SiO}_{2}$ and $v_{\mathrm{SiO}_{2}}$ - volume corresponding to one $\mathrm{SiO}_{2}$ molecule in the stress-free state [7].

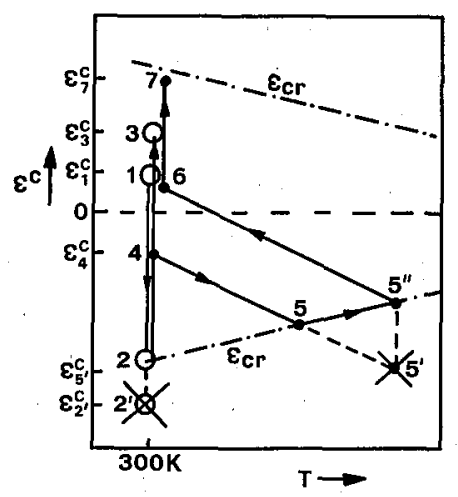

Fig. 1. Schematic draw of $\varepsilon^{c}$ changes during HP-(HT) treatment $[3,4]$. HP treatment: Sample with initial misfit $\varepsilon_{1}^{c}$ (1) under higher HP would inidicate misfit $\varepsilon_{2^{\prime}}^{c}$ (2) of negative sign [3]; however, additional defects are created at $\varepsilon^{c}=\varepsilon_{\mathrm{cr}}$ so misfit can not surpass this value. After returning sample to ambient conditions (3) misfit would be higher, $\varepsilon_{3}^{c}>\varepsilon_{1}^{c}$. HP-HT treatment: OP with initial misfit $\varepsilon_{1}^{c}$ (1) would have misfit $\varepsilon_{4}^{c}$ (4) at HP and $\varepsilon_{5}^{c}$ $\left(5^{\prime}\right)$ at HP-HT. But at $\varepsilon^{c}=\varepsilon_{\mathrm{cr}}(5)$ additional defects are created and $\varepsilon^{c}$ can not surpass this value, reaching $5^{\prime \prime}$ at HT. After returning sample to ambient conditions (7) misfit at $\mathrm{OP} / \mathrm{Si}$ boundary would be higher than that for initial sample $\left(\varepsilon_{7}^{c}>\varepsilon_{1}^{c}\right)$.

Finally it can be written $[3,4,7]$ taking into account Eqs. $(1,2)$ :

$$
\Delta a / a \propto \varepsilon^{c}\left(c_{\mathrm{O}}-c_{\mathrm{f}}\right) v_{\mathrm{SiO}_{2}}\left(4 G / 3 \kappa_{\mathrm{OP}}+1\right)-b\left(c_{\mathrm{O}}-c_{\mathrm{f}}\right) \text {, }
$$

where $b$ - coefficient in the formula for $a$ dependence on $c_{0}$ [7]. The second term in Eq. (3) relates to the precipitation and so removing the interstitial $\mathrm{O}_{i}$ atoms from the lattice. In the case of the HP treatment at room temperature the concentration of $\mathrm{O}_{i}$ does not change and this term can be neglected.

At sufficiently high HP additional defects are created at the OP/Si boundary. It can cause the increase of $\varepsilon^{c}$ (Fig. 1) and so the change of $a$ (Eq. (3)). This effect is more pronounced in the case of pulsating pressures and dependent on the higher values of $\varepsilon^{c}$ and so on the higher shear stresses [3,6]. This phenomenon is the same as the one observed during thermal oxidation of $\mathrm{Si}$ wafers and related to crushing of the $\mathrm{Si}$ protrusions at the OP/Si interface [8], moreover that in fact OP is of plate-like shape. The self interstitials and vacancies are created; because of negligible diffusion at room temperature such defects are localized at the $\mathrm{OP} / \mathrm{Si}$ interface. It influences also $\kappa_{O P}$ so its value would be higher, because of intermixing of $\mathrm{SiO}_{2}$ and $\mathrm{Si}$ under "up and down" HP cycles and so the composition of OP would be $\mathrm{SiO}_{2-x}$ instead of $\mathrm{SiO}_{2}\left(\kappa_{\mathrm{Si}}=98 \mathrm{GPa}, \kappa_{\mathrm{SiO}_{2}}=40 \mathrm{GPa}, G_{\mathrm{Si}}=68 \mathrm{GPa}\right)$. Also other parameters in Eq. (3) are influenced thus causing the change of $a$.

The "internal pressure" [2] would be also higher in the case of more extended defects with higher misfit - OP with "cloud" of self-interstitials and vacancies. It 
explains a decrease of $I_{\mathrm{A}}$ with HP. Annealing of the HP treated samples leads to the complicated processes of annihilation of self-interstitials and vacancies and/or to their diffusion into the bulk and to precipitation of the remaining $\mathrm{O}_{i}$ atoms at the new created nucleation centres. The diffusion and precipitation cause rise of the $a$ value. The value of $\left(c_{0}-c_{f}\right)$ increases because of additionally nucleated OP, the value of $\varepsilon^{\mathfrak{c}}$ would be higher if dislocation loops are created [6]. The decrease of $I_{\mathrm{A}}$ also observed after annealing supports the above conclusions. The HP-US treated samples indicate the higher value of $I_{\mathrm{A}}$ and the lower value of $a_{293 \mathrm{~K}}$ as compared to that of the samples HP treated at the same pressure. This effect is related to enhanced annihilation of point defects created during the HP treatment and corresponds to above discussed effect of additional annealing.

The phenomena in the HP-HT treated $\mathrm{Cz}-\mathrm{Si}$ samples are more complicated and dependent also on duration of the HP-HT treatment. Except the formation of "new" defects and dissolution of the previously existing ones [6] the below listed processes are of importance [4]:

1. At HP-lower HT the enhanced gettering causing decrease of $a$ and $\alpha$ as well as the $I_{\mathrm{A}}$ rise takes place.

2. For the HP-higher HT treated samples $\varepsilon^{c}$ can exceed the critical value for dislocation loops creation. For the sample returned to the ambient conditions it results in the higher value of misfit $\varepsilon^{c}$ (Fig. 1) and so of $a$ (Eq. (3)), whereas $I_{\mathrm{A}}$ remains still enhanced because of general improving of the crystal perfection.

The authors thank M.Sc. J. Zembaty for the help during experiments.

\section{References}

[1] A. Misiuk, J. Wojciechowski, J. Adamczewska, J. Jabłoński, J. Bąk-Misiuk, A. Moroz, in Proc. 32 IWK Imenau 1987, Techn. Hochschule Ilmenau, Ilmenau 1987, Vol. 4, p. 85.

[2] J. Bak-Misiuk, A. Misiuk, J. Adamczewska, J. Jabłoński, A. Moroz, A. Morawski, Acta Phys. Pol. A73, 505 (1988).

[3] J. Bąk-Misiuk, A. Misiuk, K. Klima, K. Kucharski, M. Skibska, in Defects in Crystals, Ed. E. Mizera, World Scientific, Singapore 1988, p. 359.

[4] A. Misiuk, J. Adamczewska, J. Wojciechowski, J. Bąk-Misiuk, A. Morawski, in Proc. 35 IWK Ilmenau 1990, Techn. Hochschule Ilmenau, Ilmenau 1990, Vol. 4, p. 104.

[5] Z. Witczak, Patent BRP (2485), P 271717 from 8 April 1988.

[6] J. Jung, M. Lefeld-Sosnowska, Philos. Mag. A 50, 233 (1984); J. Jung, ibid. 50, 257 (1984).

[7] V. Holý, J. Härtwig, Phys. Status Solidi B 145, 363 (1988).

[8] M. Umeno, S. Baba, T. Kojima, N. Ohmae, in Defect Control in Semiconductors, Ed. K. Sumino, Elsevier, Amsterdam 1990, p. 273. 